\title{
LA JUVENTUD COMO EARLY ADOPTER DEL CAMBIO TECNOLÓGICO Un análisis de los jóvenes aragoneses (España)
}

\author{
Rubén Ramos Antón \\ Universidad de Castilla-La Mancha, Ciudad Real, Espanha \\ David Pac Salas \\ Universidad de Zaragoza, Zaragoza, Espanha
}

Resumen El objetivo del presente artículo es analizar el papel que desarrolla la juventud en la actualidad como colectivo social que mantiene una especial relación con los avances tecnológicos. En concreto, se pretende conocer si los jóvenes aragoneses forman parte del colectivo conocido como early adopter, teniendo en cuenta las diferentes teorías sobre difusión de la tecnología. La investigación se desarrolla por medio de una metodología cualitativa, con la realización de ocho historias de vida. Los resultados de la investigación confirman el papel de early adopter de los jóvenes de la Comunidad Autónoma Aragonesa.

Palabras-clave: juventud, cambio tecnológico, early adopters, Aragón, tecnologías de la información y la comunicación.

Resumo O objectivo do presente artigo é analisar o papel que desenvolve a juventude hoje em dia como grupo social que mantém uma especial relação com os avanços tecnológicos. Especificamente, pesquisa-se conhecer se os jovens aragoneses fazem parte do coletivo conhecido como early adopter, tendo em conta as diferentes teorias sobre a difusão da tecnologia. A pesquisa desenvolve-se por meio de uma metodologia qualitativa, com a realização de oito histórias de vida. Os resultados da pesquisa confirmam o papel de early adopters dos jovens da Comunidade Autónoma de Aragão.

Palavras-chave: juventude, mudança tecnológica, early adopters, Aragão, tecnologias da informação e a comunicação.

Abstract The objective of this paper is to analyze the role that develops with the youth of today as a social collective that maintains a special relationship with technological advances. In particular, the intention is to know if the youth of Aragón form a part of the collective known as early adopters, taking into account the different theories about the diffusion of technology. The research is conducted by means of qualitative methodology gathered through eight life stories. The results of the research confirm the role of the youth from the Autonomous Community of Aragón as early adopters.

Keywords: youth, technological change, early adopters, Aragon, information and communication technologies.

Résumé Le but de cet article est d'analyser le rôle que développent les jeunes de nos jours en tant que collectif social qui mantient une relation spéciale avec les progrès technologiques. Concretement, in vise à connaître si les jeunes aragonais forment partie du collectif connu comme early adopter en tenant compte des différentes theories à propos de la difussion de la technologie. L'enquête se developpe au moyen d'une méthodologie qualitative, avec la réalisation de huit histoires de vie. Les résultats de l'enquête confirment le role d'early adopter des jeunes de la Comunité Autonome d'Aragon (Espagne).

Mots-clés: jeunesse, changement technologique, early adopters, Aragon, technologies de l'information et de la comunication. 


\section{Introducción}

Los problemas sobre la juventud se han convertido en un asunto de envergadura. Así, los jóvenes han llenado en numerosas ocasiones la agenda política, académica y pública de la sociedad occidental y española en los últimos decenios. Los problemas juveniles son habitualmente sobrerrepresentados en los medios de comunicación de masas: adicciones (drogas, alcohol), consumo masivo, hedonismo, violencia, etc.

Ante esta visión pesimista de la juventud, en nuestro caso, en la actualidad, en plena revolución digital, tras la aparición de internet y la tecnología móvil, nos interesamos por analizar el rol que desempeña la juventud como early adopter de las tecnologías de la información y la comunicación, que resulta cada vez más evidente. De hecho, la relación de los jóvenes con las tecnologías de la información ha dado lugar a conceptos como el de digital natives, generación net, generación@ o\#generación, entre otros. En la última década se han realizado numerosos estudios sobre jóvenes y nuevas tecnologías. Por ejemplo, en España, Puente et al. (2015) realizan una aproximación a los distintos enfoques. También, se han desarrollado estudios en Portugal; recientemente Campos, Pereira y Simões (2016), estudian el uso de los medios digitales en los jóvenes en Portugal y su utilización en las formas de activismo y la participación. O bien, Ponte (2011), que discute la expresión generación digital.

El objetivo principal de este texto, por lo tanto, es conocer el modo en el que los jóvenes, nativos digitales, se convierten en trendsetters (marcadores de tendencias) dentro de la sociedad, conocida su capacidad para experimentar el cambio social. ¿Cuál es el papel de definidores de tendencias (trendsetters) tecnológicas de los jóvenes aragoneses entre 20 y 30 años? La principal hipótesis del texto es que los jóvenes juegan un importante papel como definidores de tendencias tecnológicas, si bien cada vez se acortan más las distancias con las generaciones que les anteceden. La brecha digital (digital divide) tiende a reducirse en todos sus valores, también en el que hace referencia a la edad.

En este texto se presentan los resultados de una investigación que se desarrolla a través de las historias de vida. ${ }^{1}$ Para ello se trasladan los principales resultados de las biografías de los jóvenes, donde se relatan las diversas transiciones o migraciones que han ido realizando a lo largo del tiempo a través de aplicaciones, programas, dispositivos, así como su relación con las otras generaciones de su entorno.

Las conclusiones de la investigación confirman que los jóvenes se consolidan como early adopters y definidores de tendencias, que posteriormente se extienden entre otros sectores de población. Un papel que, además, se desempeña de igual modo en los jóvenes investigados provenientes del ámbito urbano y del ámbito rural.

1 Este texto tiene su base en la tesis doctoral “La segunda piel. Hábitos culturales, tecnologia y jóvenes aragoneses em la era digital” realizada por Rubén Ramos Antón y codirigida por David Pac Salas. Su defensa se realizó en junio de 2016, obteniendo la máxima calificación. 


\section{Juventud y tecnología}

Como ha ocurrido en otros momentos de la historia, en la actualidad se considera que la juventud representa una posición desde y a través de la cual se experimenta el cambio social (Urteaga, 2012: 27). La juventud, de hecho, ha participado históricamente en la difusión de los avances tecnológicos, como ocurría en las áreas rurales a partir de la industrialización. Favorecidos por la movilidad motivada por razones como la búsqueda de empleo, los estudios o el servicio militar, los jóvenes se convirtieron en "promotores de las novedades" de las ciudades en el ámbito rural (Fabre, 1996: 94).

Este papel de difusión de las innovaciones ha alcanzado una dimensión todavía más importante con la revolución tecnológica que supuso la aparición de internet y las tecnologías móviles. Mead (1971) ya señalaba hace cuatro décadas que las transformaciones tecnológicas eran causa de la ruptura generacional. A principios del siglo XXI aparece el concepto de digital natives, acuñado en primera ocasión por Prensky (2001). Este concepto englobaría a los jóvenes nacidos en torno a la primera eclosión de la era digital, en los años 90 del pasado siglo. Los digital natives surgirían como contraposición a los digital immigrants, que representarían las generaciones anteriores. En el caso de los primeros, se encontrarían perfectamente adaptados a un ecosistema que les sería propio, el del mundo digital, mientras que los segundos se verían obligados a adaptarse, lo que conllevaría que, como ocurre en el caso de las personas que migran y se deben adaptar a una nueva lengua y cultura, arrastren un acento de migrante prácticamente toda su vida.

Aparte de la de Prensky, han surgido denominaciones similares, que tienden a definir el papel de las generaciones que han crecido rodeadas por dispositivos tecnológicos digitales. Entre otras podemos señalar las de generación net, que integraría a los nacidos entre los años 1977 y 1999 (Tapscott, 1998), así como generación@ (Feixa, 2000) o el más reciente \#generación (Feixa, 2014). El denominador común, en todo caso, es la íntima relación que los jóvenes (nacidos en los últimos años del pasado siglo) mantienen con la tecnología.

La juventud, por tanto, representa un colectivo social a partir del cual la tecnología digital ha comenzado a introducirse y consolidarse en la sociedad. Siguiendo las teorías de la construcción social de la tecnología, existirían grupos sociales relevantes (Pinch \& Bijker, 2012), que estando o no organizados atribuirían un mismo conjunto de significados a un determinado artefacto. De este modo, los miembros del colectivo, en este caso los jóvenes, compartirían la interpretación y los usos de un dispositivo, algo que tiene una gran importancia a la hora de definir las funcionalidades que se le otorgan a los objetos. A lo largo de los últimos años, en los que se han sucedido la aparición y desaparición de elementos tecnológicos, hemos podido asistir a como algunas aplicaciones tecnológicas nacían con una intencionalidad y posteriormente, con el uso que los grupos sociales relevantes le otorgaban, adquirían otras connotaciones. En cierto modo pudo ocurrir con los teléfonos móviles previos a la incorporación de internet y los SMS (Short Message Service), inicialmente diseñados como un servicio marginal de la 
telefonía móvil que prácticamente terminaron convirtiéndose en una killer app ${ }^{2}$ de los primeros dispositivos, anteriores a la incorporación de internet.

Por su parte Rogers (1983) en su teoría de difusión de la innovación identifica varios grupos que intervendrían en la expansión social de cada avance tecnológico. De este modo, un colectivo crucial sería el de los early adopters, que representarían el primer grupo en adquirir cada avance tecnológico tras el colectivo, mucho más minoritario, de los pioneros. Si consideramos a los jóvenes como early adopters, estos ejercerían una función de liderazgo a través de la cual otros grupos sociales, especialmente los potential adopters, los tendrían como referencia, hasta el punto de esperar sus consejos e informaciones sobre la innovación en cuestión para terminar incorporándola en sus vidas (Rogers, 1983: 249).

Es necesario recordar que la juventud no surge como objeto de la producción cultural hasta después de la Segunda Guerra Mundial (Reguillo, 2000: 24; Martel, 2010: 53; Feixa, 2014: 80). De hecho, a pesar del papel que pudo desempeñar en otros momentos históricos, como se ha apuntado anteriormente, el presente cambio tecnológico, el protagonizado por la revolución digital, representa la primera vez en la que los jóvenes no parten de una posición subalterna (Feixa, 2014: 45). Sería por lo tanto una novedad que la juventud adoptase un papel de early adopter a la hora de dar a conocer los avances tecnológicos y que coincide con un momento histórico en el que este colectivo se ha convertido en objeto de consumo.

La tecnología forma por lo tanto a día de hoy un papel determinante en la vida de los jóvenes, y no porque esta represente un interés en sí mismo para ellos, sino porque a través de ella, consiguen estar en contacto con sus pares (Boyd en Pisani y Piotet, 2009:34). Ese manejo habitual de la tecnología como medio y forma de comunicación despierta el interés en el resto de generaciones y colectivos sociales, hasta el punto que posteriormente terminan haciendo suyos los usos que los jóvenes otorgan a las tecnologías de la información y la comunicación. En ese sentido, efectivamente, en el que se puede pensar que los jóvenes ejercen de early adopters sobre el resto de colectivos sociales.

Puente et al. (2015), en su análisis sobre juventud y nuevas tecnologías de la información y la comunicación, hacen una clasificación en relación a la temática principal de investigación: internet, telefonía móvil, redes sociales y videojuegos. Del total de estas líneas de investigación podemos señalar un sustrato teórico en dos cuestiones principales. Por un lado, la vinculación como medio social y de interacción, las diferentes dimensiones de las tecnologías de la información tienen estrechamente a internet con esa llamada sociedad de la información. Y por otra parte, la consolidación de la rotura de la barrera entre productor/consumidor y entre emisor/receptor, una idea que ya sugirió McLuhan (1996: 354) y posteriormente sería desarrollada por Cloutier (1975) a través del concepto de EMIREC. Ambas cuestiones ofrecen puntos de interés sobre los que pivota nuestra investigación.

2 Este concepto, que proviene del marketing, define una aplicación que da sentido a otro programa, tecnología o incluso a un hardware específico (Downes y Mui, 1998). 


\section{Metodología}

Para llevar a cabo los objetivos de investigación se usó una perspectiva contextual (Bergua Amores, 2004), con el objeto de conocer las interrelaciones entre las estrategias familiares, mecanismos, prácticas, etc. y, las disposiciones, decisiones, etc. que toman los jóvenes (López Montaño, 2005). Y en concreto dentro de la perspectiva contextual, se ha optado por el método biográfico. En general, esta metodología ha sido utilizada por diferentes autores. Uno de los pioneros es Bertaux y Bertaux (1993) con su trabajo sobre los panaderos. En España, podemos destacar los trabajos de Sarabia (1985), Pujadas (2002) y Sanz (2005). En Latinoamérica los estudios de Sautu (2004). En concreto, entre los estudios sobre jóvenes destacamos la investigación sobre los jóvenes madrileños de Vallés (1989), las biografías de jóvenes canarios (Cardenal de la Nuez, 2006) y las trayectorias sociales de los jóvenes de Zaragoza (Pac Salas y Ventura de Pedro, 2015). Más recientemente hemos encontrado numerosos estudios con este enfoque para asuntos juveniles: Kovács (2014) en la ciudad de Lisboa, Di Leo et al. (2013) para la ciudad de Buenos Aires o, Pulido et al. (2013) en Bogotá.

Todo ello, se concretó en la reconstrucción historias de vida a partir de la realización de entrevistas en profundidad. Esta investigación partió de la realización de ocho historias de vida a otros tantos jóvenes aragoneses de entre 20 y 30 años, a lo largo de los años 2014 y 2015. El propósito de esta investigación era conocer el modo en el que los cambios tecnológicos habían influido en la vida de los jóvenes y el papel y la penetración que, actualmente, desarrollan las tecnologías de la información y la comunicación en sus vidas.

\section{Análisis y principales resultados}

Los jóvenes de entre 20 y 30 años han tenido una relación muy familiar con la tecnología, que les ha rodeado desde su nacimiento. Los cambios producidos en los últimos años les han acompañado, con ellos han ido creciendo y desarrollándose como personas, al tiempo que las tecnologías de la información y la comunicación evolucionaban constantemente. Baste recordar que la World Wide Web, que simplificó el acceso a internet surge en 1992, año a partir del cual se comienza a popularizar la red en todo el mundo, mientras que los individuos investigados nacieron en torno a la mitad de los años 80 .

Tras la realización del estudio de campo, de los relatos de los ocho jóvenes se desprende que en muchos casos ayudaron a las generaciones que les antecedieron a descubrir, manejar e interpretar los elementos tecnológicos que comenzaban a introducirse en sus hogares.

Habitualmente se asocia a los jóvenes, los digital natives, con un especial conocimiento de las tecnologías de la información. Susca (2012: 121) se refiere a los jóvenes como los "nuevos magos, depositarios de un saber oculto". Las experiencias que ellos comentan, sin embargo, ofrecen distintas perspectivas: si por un lado, en efecto, son poseedores de un saber en cierto modo oculto, ellos mismos participan 
en hacerlo más accesible para el resto de generaciones. En otras ocasiones los elementos tecnológicos también son manejados habitualmente por sus padres, por lo que se recurre a una utilización negociada entre ambos. Así se daba, por ejemplo, con el ordenador de sobremesa en los primeros años de revolución digital, antes de la proliferación de elementos portátiles.

De este modo se comprobaría como existe una tendencia paulatina a la desaparición de la brecha digital, un término que viene a definir la diferencia que se produce en el acceso de personas (comunidades, estados, países) a las tecnologías de la información en su rutina diaria (Serrano y Martínez, 2003: 8). De hecho, también se observa en los últimos años una tendencia a la desaparición de esta brecha por motivos económicos, al menos en cuanto al acceso a los elementos tecnológicos frente a la posesión (Martín-Barbero, 2008: 30), especialmente entre los jóvenes, como también se pudo comprobar en la investigación desarrollada.

La relación con la tecnología de los jóvenes por lo tanto resulta temprana, puesto que durante buena parte de sus vidas han crecido rodeados de ella y han visto como esta ha ido evolucionando. Antes incluso de los elementos más propios de la eclosión digital (ordenadores, internet, teléfono móvil, smartphone, tablets, etc.) los jóvenes se familiarizaron con otros elementos, como los aparatos de reproducción o grabación de vídeo o DVD. Los relatos coinciden en señalar como en muchas ocasiones eran ellos los encargados de programar o utilizar estos utensilios.

En ocasiones sí [enseñaba a sus padres a programar el video] lo que pasa que muchas veces me decían “Enséñanos” a grabar, por ejemplo, que te ponías, cuando ya salieron los videos estos con programación y todo. Pues siempre te decían, “Oye, enséñanos". A lo que te ponías a enseñarles, a lo que dabas unos pasos básicos te volvían a preguntar lo del principio, un poco que acabábamos desquiciados. Entonces ya pasaba yo a prácticamente hacerlo. [i3]

Esta situación se producía de igual modo con independencia del grado de formación o instrucción de los padres y a pesar de que estos pudieran estar más familiarizados con las tecnologías de la información.

Mi madre no, que mi madre sí que tiene maña, pero a mi padre aún le ponemos nosotros el DVD. [i7]

En el caso de esta joven (i7) su padre, ingeniero industrial, estaba habituado a trabajar con ordenadores, que también utilizaba para usos destinados al ocio, de hecho manifiesta unas mayores destrezas para las descargas (películas, música, etc.) que su propia hija. A pesar de ello, recurre a sus hijos (la joven investigada y su hermano menor) para programar el DVD.

La llegada, posteriormente, de los ordenadores y con ellos de internet, supondrá un salto cualitativo en la relación con la tecnología. En el caso de los ordenadores suelen llegar de la mano de los padres/madres o personas mayores, si bien, los jóvenes de entre 20 y 30 años logran familiarizarse con ellos sin gran esfuerzo. 
Aquí el que más sabía era mi tío, que es veterinario y que por su trabajo lo empleaba y tal y entonces eso, ya se compró uno para utilizarlo para el trabajo y para uso propio. Pero era más por el trabajo ¿No? Entonces sí era el que más manejaba, incluso había hecho algún curso y tal.

Pero eso, lo que decías tú. Que nosotros desde críos pues claro, lo coges en seguida todo y en seguida pues ya dominas. Entonces eso, pues mi padre también empezó con el MacIntosh, con el más bueno, y entonces pues eso, se empezó a llevar la contabilidad por el ordenador y hacer cosas así. Ese no se podía conectar a internet y entonces se compró el de mi padre. Un PC normal. Entonces pues eso, ya automáticamente sí, creo que tal cual comprárselo se puso internet, de estos primeros que iban lentos aquí iba muy lento, muy lento, muy lento... [i1]

Pues a los diez años o por ahí, más o menos. Me acuerdo que mi padre hizo un curso de informática o algo así, entonces eran unos ordenadores nuevos que se los dejaban a mejor precio... y lo compró. Aunque luego ya ves, yo estaba todo el día jugando al buscaminas. Bueno, todo el día, que tampoco...

Al buscaminas este, que no había otro juego. Y luego lo utilizaba, un poco más mayor, para mirar cosas en internet no, porque no tenía, pero tenía enciclopedias y eso en $C D$, la Encarta... para eso. [i2]

Paradójicamente, el único caso de los jóvenes en el que la adquisición del primer ordenador responde a una iniciativa suya es el del joven migrante investigado, natural del norte de Marruecos. En su caso adquirió una computadora de segunda mano (procedente de Francia), cuando él ya contaba con casi 18 años.

Sí. Me lo compré yo, sí, sí. Me lo compré yo, sí, sí. Además me acuerdo, antes de venirme aquí [de emigrar a España]. Soy el primero que ha traído un ordenador, una torre entonces, vamos, las torres ya sabes de aquellos años, que parece que has traído una tele, ¿No? [i5]

Otro dato a tener en cuenta es que el estudio deja constancia de que en esta ocasión los avances tecnológicos llegan casi al mismo tiempo a zonas urbanas y zonas rurales, tradicionalmente más apartadas y periféricas. La desaparición o redefinición de la distancia geográfica con el desarrollo de las tecnologías de la información es una idea defendida, entre otros, por autores como Debord (2005: 144), Manovich (2005: 232) o Ramos (2016) que los jóvenes investigados confirman con sus propias experiencias. En el caso de los jóvenes que provienen del ámbito rural (i1, i2, i3 e i8), estos manifiestan una relación similar con las avances tecnológicos (bien sea ellos directamente o los jóvenes de su entorno) que en el caso de los que provienen o residen en las ciudades (i4, i5, i6 e i7).

En el caso de i1, joven procedente de un pequeño pueblo del Sobrarbe, una comarca del Pirineo aragonés, el agua corriente (potable) llegó a su casa cuando él tenía cuatro 
años. Para entonces ya disponía de teléfono fijo en su domicilio y poco después entraría en su domicilio el primer ordenador.

En otra zona distinta de Aragón, también montañosa y tradicionalmente apartada, en el Maestrazgo, otro joven (i8), recuerda que algunos de sus amigos, que habitan en masadas, ${ }^{3}$ disponen de acceso a la tecnología a través de internet y la telefonía móvil, al tiempo que carecen de conexión a la red pública de electricidad o de agua corriente.

Sí, si tengo amigos que viven por ahí, en masadas y lo tienen prácticamente todo o con un molinillo de estos de viento y eso... Lo tienen prácticamente... que les sirve, con eso.

Sí, sí, todo. Hay un amigo que tengo, pues mucho el... pedir pide muchísimas cosas por internet, casi todo lo encarga por internet, llegar ahí no sé si se lo traen allí y lo dejarán aquí, tiene familia en Alcañiz y no sé si lo dejarán aquí y se lo subirán después y eso, pero, por ejemplo, también ha estudiado mecánica y eso y para los recambios de los coches y todo lo pide todo por internet. Todo.

[...] Ése sí que es manitas y de hacer inventos y de todo... "Pues he visto por internet esto, lo he pedido, a ver si me llega". Ese sí que lo pide todo por internet. Y vive allí, en una masada, que no tiene luz ni nada, con el generador y con las placas y de todo. Placas sí que tiene unas cuantas y molinillo y de todo. [i8]

Los testimonios también coinciden en señalar que la forma en la que aprendieron a relacionarse con las tecnologías de la información fue mayoritariamente a través de sus pares. A pesar de que en sus años de formación escolar ya se incorporó la informática, una incorporación que se criticó en los primeros años de revolución digital por tardía o poco adaptada a las aceleradas transformaciones que estaba viviendo la sociedad (Castells, 2001: 286-287). La alfabetización mediática vino, por lo tanto, de la mano de videojuegos, de la multiplicidad de pantallas, como señala Tapscott (1998) hace ya casi dos décadas, un tipo de aprendizaje más propio de autodidactas (Marta-Lazo y Gabelas, 2011: 39), que el que tuvieron sus padres, para los que en muchos casos, los jóvenes ejercen de auténticos cicerones tecnológicos.

Tanto en el caso de las áreas urbanas como de las rurales, entre jóvenes con mayor o menor poder adquisitivo, si existe un elemento tecnológico del que los jóvenes se han apropiado ha sido el smartphone. Se trata del teléfono en el que convergen diferentes aplicaciones y que supone una auténtica revolución comunicativa, desde el momento en el que tiene acceso a internet. Una apropiación entendida como adaptación de los medios o tecnologías de la información y el conocimiento a sus propias necesidades (Morales Álvarez y Loyola, 2011: 142-143) y que forma parte de un proceso de consumo cultural en el que el valor simbólico prevalece sobre los valores de uso y de cambio (García Canclini, 1993: 34).

3 Masadas, masías: tipo de residencia propia de la zona, tradicionalmente alejadas de los núcleos urbanos. 
A diferencia de los ordenadores de sobremesa, introducidos mayoritariamente por los padres, los siguientes elementos tecnológicos (entre ellos los ordenadores portátiles, teléfonos móviles, tablets, smartphones, etc.) responden a un interés decidido de los jóvenes. Ya convertidos, por lo tanto, en población objeto de consumo. Habitualmente los jóvenes experimentan migraciones de unos elementos tecnológicos a otros o bien, dentro de cada dispositivo, de unas aplicaciones o programas a otros.

De este modo, en sus testimonios relatan un itinerario muy similar que les hace comenzar por chats o foros para pasar a programas como el Messenger, redes sociales como Tuenti, para posteriormente utilizar de forma masiva otras, como es el caso de Facebook o de aplicaciones como Whatsapp. El denominador común de estos cambios, en muchos casos producidos en muy poco tiempo, es la presión social (Lahire, 2006: 499) a la que se ven sometidos por parte del grupo de pares (especialmente grupos de amigos, pareja, etc.).

Es a través de los smartphones donde los jóvenes han encontrado un mayor sentido a la tecnología, especialmente como medio para relacionarse con sus pares, tal y como se exponía en la introducción. Si los teléfonos móviles de primera generación tuvieron a los SMS (Short Message Service) como killer app (Downes y Mui, 1998), en el caso de los smartphones la aplicación más parecida, que alcanza un papel central, hasta el punto de casi dar sentido a todo el dispositivo es el Whatsapp. Este servicio de mensajería instantánea centraría la mayor parte de la actividad tecnológica tanto de los jóvenes investigados como de todas sus amistades. La relación con la tecnología como medio para mantener la comunicación llega hasta tal punto que los jóvenes reconocen en muchos de los casos una abierta dependencia.

Esto es una dependencia fruto del modo de vida en el que llevamos, del sistema social y económico en el que estamos involucrados y en el que nos requiere, prácticamente tener este tipo de cacharros para estar conectados y para relacionarnos con la gente. [i4]

En el transcurso de la investigación (a lo largo de los años 2014 y 2015) tan solo en un caso (i1) uno de los jóvenes no disponía de esta aplicación, lo que, a su vez, reconoció que le proporcionaba problemas de sociabilidad con sus pares, ya que era la excepción de su grupo de amigos. ${ }^{4}$

Por ejemplo yo, empecé a darle al Kayak y eso, no somos... Hay muy poca gente que le pega, aunque cada vez hay más, pero claro, cuando empiezas tienes que ir con gente, no puedes ir a lo bravo. Y entonces está muy bien lo del grupo (de Whatsapp) del Kayak, pero claro, yo no lo tengo, y claro, yo si no es de alguien que me comenta, "en el grupo del Kayak hemos puesto a ver si os apetece quedar el miércoles, tal". Pero claro, si a mí no me lo dicen pues no me entero. [i1]

4 En la actualidad la mensajería instantánea es la actividad online más habitual entre los jóvenes españoles, Urueña (2017: 24). 
El resto, incluso aquellos que mostraron menos interés por las tecnologías (como es el caso de i6 o i8), reconocieron un alto nivel de utilización de esta aplicación, incluso llegado el momento, de abierta dependencia.

El caso de la joven oscense i6 resulta paradigmático, dado que se trata de una de las que menos interés demuestra por la tecnología, incluso por la posesión de los smartphones. De hecho, los ha perdido en varias ocasiones, siendo obligada por sus padres a adquirir un dispositivo o tener un mayor cuidado de ellos. Sus padres, por otro lado, se encuentran muy familiarizados por la tecnología, a pesar de lo que ella reconocía un mayor dominio sobre el Whatsapp.

[Nivel de penetración tecnológica de la madre] en Whatsapp no, porque en Whatsapp yo ahora estoy bastante metida. Pero mi madre también está pillándole el ritmo, a lo del Whatsapp, con esto del iPhone, que es muy cómodo, sí. [i6]

La brecha digital en lo que respecta a los últimos elementos tecnológicos parece en franco retroceso, a tenor de los testimonios de los jóvenes. Al igual que los jóvenes se han apropiado del smartphone y del Whatsapp como uno de sus hábitos tecnológicos imprescindibles en sus vidas, con el tiempo esta misma situación se está produciendo en las generaciones que los anteceden. Así lo demuestra el hecho de que una buena parte de los jóvenes entrevistados (i2, i4, i6, i8) formaban parte de grupos de Whatsapp en el que coinciden, al menos con su familia nuclear.

Un aspecto que llama la atención en cuanto a la relación con sus familiares, es que los jóvenes que contaban con hermanos menores (i3, i4, i6, i7 e i8) reconocían abiertamente que estaban mucho menos familiarizados con las nuevas tecnologías que ellos. En cierto modo consideraban a los más jóvenes todavía más digital natives de lo que podían ser ellos, por lo que se repetía la brecha que ellos mantenían con sus padres. Una de las características en las que más reconocen diferenciarse es, por ejemplo, en la capacidad para escribir a través de la mensajería instantánea.

Pero sí que es verdad que mi hermana, por ejemplo, lo veo un montón, que escribe que es un pasada... A lo corto, a lo corto... Es más joven que yo. Yo creo que ese, en esa franja de edad, también se va notando un poco, que yo puedo escribir alcorzando, o sea, acortando las letras y demás... Que te quiero decir, yo lo hago pero noto como que los que vienen de detrás aún se ha incrementado o se ha posicionado más, no sé. [i3]

Otro joven (i8) reconoce que sus dos hermanas menores dedican mucho más tiempo a la tecnología de lo que lo hace él, tanto con el ordenador de casa como con los smartphones, tanto para actividades de ocio como para comunicarse con sus amigos y amigas.

El caso de i5, no obstante, resulta más complicado de evaluar, puesto que al encontrarse fuera de su ámbito familiar, no conoce exactamente el grado de penetración de las tecnologías de la información en las vidas de sus hermanos actualmente. Sin embargo, se mantiene en contacto diario con su familia (padres y hermanos) a través de la tecnología, mediante programas como Skype.

Los usos tecnológicos de los jóvenes, por lo tanto, se abren paso entre las generaciones que les preceden, si bien, retomando una idea expresada en la introducción, 
el interés manifestado por los jóvenes no es la tecnología en sí misma, sino la necesidad de mantener el contacto con sus pares. Ninguno de los jóvenes mostró un interés especial en aspectos relacionados con la programación o un conocimiento de la informática que no fuera a nivel de usuario. Por ello se podría establecer una clara diferencia entre el joven como early adopter o digital native, como usuario familiarizado de un modo casi natural con un entorno digital, y el geek o persona especialmente interesada por un tema, en este caso la informática, que seguiría siendo un colectivo mucho más reducido que el primero.

Mención aparte merece el hecho de que para el acceso a según qué contenidos (como por ejemplo las descargas de material audiovisual - música, cine, series, etc.) se puedan establecer dos simples categorías entre los jóvenes investigados: los individuos más familiarizados con la tecnología, que consiguen estos productos directamente (asumen un papel activo) y los que acceden a estos contenidos de forma secundaria (por ejemplo, a través de amistades). En ambos casos se comparte una actividad que McLuhan (1996: 292) calificó de recolector de información, un ser humano nómada, en busca en este caso de otro tipo de alimentos, lo que también evoca las palabras de Bauman sobre una sociedad en constante movimiento (2002: 137).

Yo no (descargar contenidos audiovisuales). Sí veo series (The Walking Dead), pero yo no lo descargo, pero con el chico que lo veo lo descarga él para verlos. [i2]

En otros casos el tipo de consumo de los productos culturales se realiza de forma diferente entre los jóvenes y sus padres. Mientras que los segundos preferían descargar los contenidos, los primeros optaban por el consumo en streaming, una práctica que ya en el momento de realizarse el estudio de campo de la investigación se encontraba en pleno auge.

Mi padre sí [descarga productos audiovisuales], porque mi padre no se mete en seriesli. Entonces las películas que quiere se las descarga. Y porque le gusta, porque le apetece, porque le gusta descargarse cosas. Yo no, porque yo como las veo en seriesli o en Youtube o... Es que tampoco me hace falta. [i7]

A este respecto, lo que el estudio de campo deja patente es que, efectivamente, los usos tecnológicos de los jóvenes suelen ser más avanzados que los de sus padres. Se comportan como un objeto de consumo de la tecnología, que forma parte de su cultura asociada a la idea de la construcción de su identidad (Reig y Vílchez, 2013: 102) y la construcción de espacios para encontrarse con sus pares (Boyd, 2007). A diferencia de lo que puede ocurrir en otras culturas o en otros momentos históricos, en los que se ha hablado de la cultura de la habitación (Livingstone, 2007), los actuales dispositivos permiten la movilidad y la ubicuidad del acceso a internet y la comunicación o participación en sus redes de amistades.

Sin embargo, las vivencias de los individuos investigados hacen pensar que las diferencias con los adultos tienden a ir despareciendo y que estos últimos también se han apropiado de los elementos tecnológicos y/o aplicaciones 
que parecían reservados a los jóvenes en un primer momento de la presente revolución tecnológica.

\section{Conclusiones}

Los testimonios de los jóvenes, a través de sus historias de vida, vienen a confirmar que se trata de un colectivo muy relacionado con la tecnología a lo largo de su experiencia vital. No en vano, han crecido al mismo tiempo que se sucedían los cambios tecnológicos (aparición de internet, de las tecnologías móviles, convergencia de ambas, etc.).

Tomando los conceptos expuestos en la introducción, la investigación confirma el papel relevante del que disponen los jóvenes de entre 20 y 30 años en los cambios tecnológicos. Unos jóvenes crecidos entre pantallas, como señala Tapscott, y que, efectivamente, se desenvuelven con las tecnologías de la información como en su propio ecosistema. Sin entrar a valorar si el término de digital natives resulta el más apropiado para definir la relación entre los jóvenes y la tecnología, lo cierto es que sus relatos confirman que esta supone una dimensión importante en sus vidas, especialmente a la hora de socializarse y construir su identidad personal. En ese sentido juega un importante rol la aparición de los smartphones, como elementos que son adquiridos respondiendo a su iniciativa y en los que en un primer momento manifestaron mejores destrezas que sus padres.

Sin embargo, la tendencia parece indicar que las generaciones que les preceden (sobre todo sus padres y madres) van adquiriendo paulatinamente los conocimientos básicos para desenvolverse en algunos de los entornos con los que los jóvenes se encuentran perfectamente adaptados. Es el caso, por ejemplo, de la mensajería instanéna (Whatsapp) como aplicación central del smartphone. La apropiación de otras generaciones de las tecnologías de la información y la comunicación, hace pensar si estas van a arrastrar el acento migrante, como planteaba Prenksy en 2001 o si por el contrario van a terminar adaptándose a ellas, hasta el punto de interiorizarlas totalmente. Los resultados invitan a pensar que todavía es pronto para extraer conclusiones a este respecto. Las personas que no son consideradas como digital natives, esto es, las nacidas con anterioridad a la mitad de los años 80 , han vivido los cambios tecnológicos de un modo diferente a los digital natives, puesto que no nacieron rodeados de pantallas ni en un entorno "digital". Sin embargo, los testimonios de los jóvenes señalan cómo la diferencia entre unos y otros, la llamada brecha digital (en este caso de tipo generacional) tiende a estrecharse.

Por otra parte, el análisis de los resultados sí confirma la existencia de diferentes grupos que de un modo más o menos homogéneo incorporan los avances tecnológicos a sus vidas. Se trataría, por lo tanto, de la confirmación de la existencia de los grupos sociales relevantes planteados por Pinch y Bijker. En este caso uno de los grupos sería el de los jóvenes, que otorgaría sentido a una tecnología (smartphone) y una aplicación (mensajería instantánea) como elementos para la socialización. A su vez, este grupo cumpliría una función de early adopter, concepto planteado por Rogers en su teoría de la difusión de la tecnología. De los usos que este grupo de early 
adopters realizan de la tecnología toman muy buena nota otros grupos sociales ( $p o-$ tential adopters) que podrían encarnar sus padres y familiares para terminar o no apropiándose, en una segunda fase, de estas tecnologías.

\section{Referencias bibliográficas}

Bauman, Zygmunt (2002), Modernidad Líquida, Buenos Aires, Fondo de Cultura Económica.

Bergua Amores, José Ángel (2004), “Investigación social y anamnesis: más allá de la perspectiva dialéctica", revista Papers, 72, pp. 11-30.

Bertaux, Daniel, y Isabelle Bertaux (1993), "Historias de vida del oficio de panadero", en José Miguel Marinas y Cristina Santamaría (orgs.), La Historia Oral. Métodos y Experiencias, Madrid, Debate, pp. 231-250.

Boyd, Dannah (2007), “Why youth (heart) social network sites: the role of networked publics in teenage social life", en David Buckingham (org.), Youth, Identity and Digital Media, Cambridge, MA, The MIT Press.

Campos, Ricard, Inês Pereira, y José Alberto Simões (2016), “Ativismo digital em Portugal: um estudo exploratório", Sociologia, Problemas e Práticas [en línea], 82 I 2016, puesto en línea el 12 octubre 2016, consultado el 08 febrero 2017, URL: http://spp.revues.org/2460

Cardenal de la Nuez, María Eugenia (2006), El Paso a la Vida Adulta. Dilemas y Estrategias ante el Empleo Flexible, Madrid, Centro de Investigaciones Sociológicas.

Castells, Manuel (2001), La Galaxia Internet, Madrid, Areté.

Cloutier, Jean (1975), L'Ere d'Emerec ou la Communication Audio-Scrito-Visuelle à l'Heure des Self-Media, Montreal, Les Press de l'Université de Montréal.

Debord, Guy (2005), La Sociedad del Espectáculo, Valencia, Pre-Textos, segunda reimpresión, segunda edición.

Di Leo, Pablo Francisco, Ana Clara Camarotti, Martín Güelman, y María Cecilia Touris (2013), “Mirando la sociedad a escala del individuo: el análisis de procesos de individuación en jóvenes utilizando relatos biográficos", revista Athenea Digital, 13 (2), pp. 131-145.

Downes, Larry, y Chunka Mui (1998), Unleashing the Killer App. Digital Strategies for Market Dominances, Boston, Harvard Business School Press.

Fabre, Daniel (1996), "Forjar la juventud en el pueblo", en Giovanni Levi y Jean Claude Schmitt (directores), Historia de los Jóvenes. II. La Edad Contemporánea, Madrid, Taurus, Pensamiento, pp. 61-100.

Feixa, Carles (2000), “Generación @. La juventud en la era digital”, revista Nómadas, 13, pp. 75-91.

Feixa, Carles (2014), De la Generación@ a la \#Generación, Barcelona, Ned.

García Canclini, Néstor (1993), "El consumo cultural y su estudio en México: una propuesta teórica", en Néstor García Canclini (coord.), El Consumo Cultural en México, México, DF, Conaculta, pp. 15-42.

Kovács, Ilona (2014), “Trayectorias laborales y de vida de jóvenes: un análisis cualitativo", revista Sociología del Trabajo, 80, pp. 28-49. 
Lahire, Bernard (2006), La Culture des Individus. Dissonances Culturelles et Distinction de Soi, París, La Découverte/Poche.

Livingstone, Sonia (2007), “From family television to bedroom culture: young people's media at home", en Eoin Devereux (org.), Media Studies: Key Issues and Debates, Londres, Sage, pp. 302-321.

López Montaño, Luz María (2005), “El cómo en la investigación de familia: reflexiones de la experiencia desde un abordaje cualitativo", Revista Latinoamericana de Ciencias Sociales, Niñez y Juventud, 3 (1), pp. 213-236.

Manovich, Lev (2005), El Lenguaje de los Nuevos Medios de Comunicación. La Imagen en la Era digital, Barcelona, Paidós Comunicación.

Marta-Lazo, Carmen y José Antonio Gabelas (2011), “Hacia la multialfabetización digital de los jóvenes en red", en Estrella Martínez y Carmen Marta-Lazo (coords.), Jóvenes Interactivos, Oleiros (A Coruña), Netbiblo, pp. 37-52.

Martel, Frédéric (2010), Cultura Mainstream. Cómo Nacen los Fenómenos de Masas, Madrid, Taurus.

Martín-Barbero, Jesús (2008), “El cambio en la percepción de los jóvenes: socialidades, tecnicidades y subjetividades", en Roxana Morduchowicz (coord.), Los Jóvenes y las Pantallas. Nuevas Formas de Sociabilidad, Buenos Aires, Gedisa, pp. 25-46.

Mead, Margaret (1971), Cultura y Compromiso. Estudio sobre la Ruptura Generacional, Buenos Aires, Granica.

McLuhan (1996), Comprender los Medios de Comunicación. Las Extensiones del Ser Humano, Barcelona, Paidós.

Morales, Susana, Alejandro Álvarez, y María Inés Loyola (2011), “Apropiación de tecnologías de la información y la comunicación e interactividad juvenil: realidades y desafíos", en Estrella Martínez y Carmen Marta-Lazo(coords.), Jóvenes Interactivos, Oleiros (A Coruña), Netbiblo, pp. 139-154.

Pac Salas, David, y Ventura de Pedro, Tirso (2015), “Trayectorias de jóvenes de Zaragoza (España) en configuraciones familiares obreras", Revista Latinoamericana de Ciencias Sociales, Niñez y Juventud, 13 (2), Colombia.

Pinch, Trevor, y Wiebe Bijker (2012), “The social construction of facts and artifacts: or how the Sociology of Science and the Sociology of Technology might benefit each other", en Wiebe Bijker, Thomas Hugues, y Trevor Pinch, (orgs.), The Social Construction of Technological Systems, Cambridge, MA, The MIT Press, pp. 11-44.

Pisani, Francis, y Dominique Piotet (2009), La Alquimia de las Multitudes. Cómo la Web Está Cambiando el Mundo, Barcelona, Paidós.

Ponte, Cristina (2011), “Uma geração digital? A influência familiar na experiência mediática de adolescentes", Sociologia, Problemas e Práticas [en línea], 65 | 2011, puesto en línea el 14 marzo 2012, consultado el 08 febrero 2017, URL : http://spp.revues.org/94

Prensky, Marc (2001), “Digital natives, digital inmigrants”, revista On the Horizon, MCB University Press, 9 (5), pp. 1-6.

Puente, Héctor, Marta Fernández, Costán Sequeiros, y Mélida López (2015), “Los estudios sobre jóvenes y TICs en España”, revista Juventud, 110, pp. 155-172.

Pujadas, Juan José (2002), El Método Biográfico. El Uso de las Historias de Vida en Ciencias Sociales, Madrid, Centro de Investigaciones Sociológicas. 
Pulido, Sandra, Juliana Castro-Osorio, Marlyn Peña, y Diana Paola Ariza-Ramírez (2013), "Pautas, creencias y prácticas de crianza relacionadas con el castigo y su transmisión generacional", Revista Latinoamericana de Ciencias Sociales, Niñez y Juventud, 11 (1), pp. 245-259.

Ramos, Rubén (2016), “Penetración tecnológica en Aragón: de periferia a centro. Technological penetration in Aragon: from periphery to center", revista F@ro, 24 (2), pp. 143-167.

Reguillo, Rosana (2000), Emergencia de Culturas Juveniles. Estrategias del Desencanto, Bogotá, Grupo Editorial Norma.

Reig, Dolors, y Luis Fernando Vílchez (2013), Los Jóvenes en la Era de la Hiperconectividad. Tendencias, Claves y Miradas, Madrid, Fundación Telefónica / Fundación Encuentro.

Rogers, Everett (1983), Diffusion of Innovations, Nueva York, The Free Press, tercera edición.

Sanz, Alexia (2005), “El método biográfico en investigación social: potencialidades y limitaciones de las fuentes orales y los documentos personales", revista Asclepio, 57 (1), pp. 99-115.

Sarabia, Bernabé (1985), “Historias de vida”, Revista Española de Investigaciones Sociológicas, 21: 165-186.

Sautu, Ruth (comp.) (2004), El Método Biográfico. La Reconstrucción de la Sociedad a Partir del Testimonio de los Actores, Buenos Aires, Ediciones Lumiere.

Serrano, Arturo, y Evelio Martínez (2003), La Brecha Digital. Mitos y Realidades, México, DF, Editorial UABC.

Susca, Vincenzo (2012), Deleite Trágico. Las Formas Elementales de la Vida Electrónica, Barcelona, Península, Atalaya.

Tapscott, Don (1998), Growing up Digital. The Rise of the Net Generation, Nueva York, McGraw-Hill.

Urteaga, Maritza (2012), “De jóvenes contemporáneos: trendys, emprendedores y empresarios culturales", en Néstor García Canclini, Francisco Cruces, y Maritza Urteaga (coords.), Jóvenes, Culturas Urbanas y Redes Digitales, Barcelona, Ariel, pp. 25-44.

Urueña, Alberto (coord.) (2017), La Sociedad en Red. Informe Anual 2016, Madrid, Ministerio de Hacienda, Turismo y Agenda Digital, Gobierno de España.

Vallés, Miguel (1989), Abrirse Camino en la Vida. Proyectos Vitales de Jóvenes Madrileños, tesis doctoral, Universidad Complutense de Madrid.

Rubén Ramos Antón. Profesor ayudante doctor. Facultad de Comunicación. Universidad de Castilla-La Mancha.E-mail: Ruben.Ramos@uclm.es

David Pac Salas. Profesor titular de Sociología. Facultad de Economía y Empresa. Universidad de Zaragoza. E-mail: davidpac@unizar.es

Receção: 09 de janeiro de 2018 Aprovação: 25 de janeiro de 2018 
